\title{
Acesso aos Serviços de Saúde: o que dizem as mulheres privadas de liberdade?
}

\author{
Access to Health Services: What the Women Deprived of Their Liberty?
}

\author{
Márcia Vieira dos Santos ${ }^{1}$ \\ Valdecyr Herdy Alves ${ }^{2}$ \\ Audrey Vidal Pereira ${ }^{3}$ \\ Diego Pereira Rodrigues ${ }^{4}$ \\ Giovanna Rosário Soanno Marchiori ${ }^{5}$ \\ Juliana Vidal Vieira Guerra ${ }^{6}$
}

\footnotetext{
${ }^{1}$ Enfermeira. Mestre em Saúde Materno-Infantil. Sistema Penitenciário do Estado do Rio de Janeiro, Brasil. E-mail: enfa.marcia@oi.com.br.

2 Enfermeiro. Doutor em Enfermagem. Professor Titular da Escola de Enfermagem Aurora de Afonso Costa da Universidade Federal Fluminense. Rio de Janeiro, Brasil. E-mail: herdyalves@yahoo.com.br.

${ }^{3}$ Enfermeiro. Doutor em Saúde Pública. Professor Adjunto da Escola de Enfermagem Aurora de Afonso Costa da Universidade Federal Fluminense. Rio de Janeiro, Brasil. E-mail: auviprof@yahoo.com.br.

${ }^{4}$ Enfermeiro. Mestre em Enfermagem. Professor Adjunto do Centro Universitário Anhanguera de Niterói do Curso de Enfermagem. Rio de Janeiro, Brasil. E-mail: diego.pereira.rodrigues@gmail.com. Autor correspondente.

5 Enfermeira. Mestre em Saúde Materno Infantil. Professora Adjunta da Faculdade Novo Milênio, Espírito Santo, Brasil. E-mail: giovanna_marchiori@yahoo.com.

${ }^{6}$ NUtricionista. Mestre em Saúde Materno Infantil. Universidade Federal Fluminense. Rio de Janeiro, Brasil. E-mail: julianavvguerra@yahoo.com.br.
} 


\section{Resumo}

Objetiva-se analisar como as mulheres encarceradas percebem o acesso aos serviços de saúde. Estudo descritivo, exploratório e qualitativo realizado com quarenta mulheres em um presídio feminino do Estado do Rio de Janeiro, Brasil. Foi utilizada na coleta de dados, a entrevista semiestruturada, e posterior transcrição na integra. As entrevistas foram submetidas à análise de conteúdo na modalidade temática. Antes e durante o encarceramento, as mulheres referiram dificuldades para acessar os serviços de saúde. Entretanto, surgem falas de modo contraditório, inferindo a possibilidade de, após a entrada no sistema penal, essas mulheres conseguem acessar alguns serviços de saúde jamais visitados antes do período de confinamento. Conclui-se que para garantir a aplicabilidade da política vigente, existe a necessidade de estratégias de atenção integral à saúde dessas mulheres, que assegurem o acesso aos serviços de saúde numa perspectiva ampliada de promoção, prevenção, tratamento e reabilitação, antes, durante e depois da prisão.

Palavras-chave: Acesso aos Serviços de Saúde; Assistência Integral à Saúde; Mulheres; Prisões.

\section{Abstract}

The aim is to analyze how incarcerated women perceive access to health services. This descriptive, exploratory and qualitative study was carried out with 40 women in a female prison in the state of Rio de Janeiro, Brazil. It was used in data collection, semi-structured interview, and subsequent transcription in the integrated. The interviews were submitted to content analysis in the thematic modality. Before and during incarceration, women reported difficulties in accessing health services. However, they appear contradictory, inferring the possibility of, after entering the penal system, these women were able to access some health services never visited before the confinement period. It concludes that to ensure the applicability of the current policy, there is a need for comprehensive health care strategies for these women, which ensure access to health services in a broader perspective of promotion, prevention, treatment and rehabilitation, before, during and after imprisonment.

Keywords: Access to Health Services; Integral Health Assistance; Women; Prisons. 
Introdução

A política pública em saúde, voltada para o sistema penitenciário, foi estabelecida em 1984 na Lei de Execução Penal ${ }^{(1)}$, na qual foi previsto os atendimentos médico, farmacêutico e odontológico para pessoas privadas de liberdade. Em 1988, com a Constituição Federal, art. 196, ficou assegurada a saúde como um direito de todos e dever do Estado ${ }^{(2)}$. Garantido assim, por meio de políticas públicas, a descentralização dos serviços de saúde, atendimento integral com prioridade para atividades preventivas e participação da comunidade nas ações de saúde. A complexidade do conceito ampliado de saúde, tendo em vista os aspectos relacionados à alimentação, educação, trabalho, ambiente, lazer e etc., deve embasar a operacionalização de ações assistenciais e preventivas, numa perspectiva relacionada à garantia à equidade e universalidade de acesso aos serviços de saúde ${ }^{(3)}$.

Entretanto, quando se fala de acesso à saúde torna-se necessário refletir que acessar o sistema de saúde vai para além da entrada do usuário nos serviços. $\mathrm{O}$ acesso, para ser garantido totalmente, deve-se levar em consideração questões que vão além dos atendimentos/consultas individuais. Ou seja, as demandas devem ser atendidas de modo integral, assegurando a continuidade da atenção à saúde ${ }^{(4)}$. $\mathrm{O}$ acesso à saúde, numa perspectiva de equidade, deve se remeter à ações intersetoriais das políticas públicas de saúde voltadas para educação, habitação, melhor distribuição de renda e o fortalecimento da cidadania ${ }^{(5)}$.

Desta forma, pode-se observar a existência de inúmeros fatores que interferem na garantia desse processo: renda da população, conhecimento e confiança do usuário no sistema de saúde, predisposição das pessoas em utilizar o serviço de saúde, meio disponível e tempo para acessar as ações e atividades do serviço; fonte de informação e organização da instituição de saúde, tempo/demora do atendimento, falta de recursos humanos, dificuldade de prosseguimento da atenção à saúde e necessidades diferenciadas de saúde. Tais situações contribuem para (des)qualificar ou tornar (des)igual o acesso da população à saúde ${ }^{(4-5)}$.

Efetivar o acesso em saúde com equidade e universalidade ainda é um grande desafio, pois o conceito pode se modificar conforme as necessidades, demandas e usos dos serviços de saúde de cada território e/ou grupo populacional( ${ }^{(6)}$. Assim, torna-se necessário impactar nos serviços de saúde, implicando em novas condutas e perspectivas de políticas públicas direcionadas às mulheres privadas de liberdade ${ }^{(7)}$.

Conforme a compreensão e necessidades das pessoas que usam o Sistema Único de Saúde (SUS), o acesso aos serviços (ou a falta dele) pode espelhar um processo de desigualdades características de determinados grupos vulneráveis na sociedade ${ }^{(8)}$.

Portanto, o acesso ao serviço de saúde engloba um entendimento multidimensional relacionado com políticas públicas para além das específicas relacionadas à saúde, deve concretizar a existência de serviços resolutivos que atendam à gama de complexidade da saúde, respeitando as necessidades da população ${ }^{(9)}$.

Assim, com relação ao sistema penitenciário, desde a década de 90 foi necessário um processo de adequação em relação ao SUS para garantir e ampliar o direito à saúde das pessoas privadas de liberdade. Tais mudanças foram observadas a partir de 2002, quando se percebeu um avanço na legislação através do Plano Nacional de Saúde do Sistema Penitenciário (PNSSP) cuja finalidade foca na organização da saúde penal com base nos princípios do SUS ${ }^{(10-11)}$.

Em 2014, houve nova mudança na política de saúde do sistema penal sendo instituída a Política Nacional de Atenção Integral à Saúde das Pessoas Privadas de Liberdade no Sistema Prisional (PNAISP). O objetivo geral dessa política é o de garantir, às pessoas privadas de liberdade, o acesso ao cuidado integral no SUS, respeitando-se os preceitos dos direitos humanos e de cidadania ${ }^{(12)}$.

Com o objetivo de ampliar os direitos à saúde da população feminina privada de liberdade, também em 2014, foi instituída a atual Política Nacional de Atenção às Mulheres em Situação de Privação de Liberdade e Egressas do Sistema Prisional - PNAMPE ${ }^{(13)}$. Esta política veio para reformular as práticas do sistema prisional brasileiro em relação aos direitos das mulheres, promovendo, pactuando e incentivando ações integradas e intersetoriais destinadas à complementação e ao acesso aos direitos fundamentais previstos na Constituição Federal e na Lei de Execução Penal|(13). 
A PNAMPE garante, à mulher privada de liberdade, o acesso à saúde e, em consonância com a Política Nacional de Atenção Integral à Saúde da Mulher (PNAISP), assistência à educação, à assistência jurídica e religiosa, acesso à atividade laboral, compatibilidade das horas diárias de trabalho e estudo, além da compatibilidade da atividade laboral com a condição de gestante e mãe, assegurada a remuneração, a remissão e a licença-maternidade ${ }^{(13)}$. Assim, o estudo tem o propósito de responder o seguinte questionamento: Como se configura o acesso das mulheres no sistema prisional?

Desse modo, o estudo teve como objetivo analisar como as mulheres encarceradas percebem o acesso aos serviços de saúde.

\section{Método}

Pesquisa descritiva, exploratória, com abordagem qualitativa, realizada no período de outubro de 2014 a janeiro de 2015 em uma penitenciária feminina localizada na Região Oeste do Estado do Rio de Janeiro, Brasil.

Por meio de entrevistas semiestruturadas individuais, participaram do estudo 40 mulheres privadas de liberdade, que foram identificadas a partir de uma lista nominal oficial da instituição que totalizava 358 mulheres.

A inclusão das entrevistadas ocorreu por meio dos seguintes critérios: mulheres que detinham numeração de prontuário durante o período de coletas de dados e que faziam o acompanhamento/consulta/atendimento.

Enquanto os critérios de exclusão estabelecidos foram: dificuldades físicas e psíquicas, eventual proibição das agentes penitenciárias de plantão, transferência para outra unidade do sistema prisional e saída do sistema por motivo de liberdade durante o processo de coleta de informações.

A penitenciária pesquisada é exclusiva para mulheres. Foi inaugurada em 1942, sendo a primeira prisão feminina do Estado inserida num complexo prisional que engloba 27 unidades sob o gerenciamento da Secretaria de Administração Penitenciária do Estado do Rio de Janeiro. Nesta penitenciária existe: um ambulatório de saúde, uma escola estadual de ensino regular que contempla desde a alfabetização até o ensino médio, diversas oficinas de trabalho com cursos profissionalizantes, e também existe trabalho remunerado para algumas presas, denominado faxina. Para cada três dias trabalhados, um dia é descontado de sua pena. A unidade prisional também oferece encontros religiosos de diversas crenças. Anexo à instituição estudada, existe a Unidade Materno Infantil (UMI), onde ficam as mulheres em período pós-parto com seus filhos recém-nascidos, até os seis meses de idade.

As entrevistas foram realizadas no ambulatório da penitenciária, cujo espaço físico possui consultórios, sala de procedimento, pequeno almoxarifado, banheiros para os profissionais e para as usuárias, e um corredor com uma maca. As pacientes que são atendidas neste ambulatório possuem um prontuário de saúde que fica arquivado em um armário exclusivo para este serviço. Vale lembrar que este ambulatório fica aberto somente quando um profissional de saúde se encontra trabalhando na unidade prisional.

As entrevistas aconteceram em uns dos consultórios deste ambulatório. Para iniciar cada encontro, a pesquisadora apresentava o objetivo do estudo e garantia total de anonimato às participantes. Importante destacar que para ser autorizada a realização da pesquisa no sistema prisional, a Secretaria de Administração Penitenciária do Estado do Rio de Janeiro exigiu o cumprimento de algumas questões para a coleta dos dados, como: a entrada da pesquisadora no presídio só poderia ocorrer após realização da conferência cotidiana das presas; a saída da pesquisadora da instituição teria que ser realizada uma hora antes da conferência realizada no final do dia; as gravações de áudio e filmagem, fotografias e a reprodução de qualquer documento, bem como as assinaturas do Termo de Consentimento Livre e Esclarecido (TCLE) e/ou qualquer forma de identificação nominal das pessoas privadas de liberdade foram proibidas.

A participação das mulheres ocorria após convite e aceite para integrar à pesquisa. Cabe destacar que o Estado era o responsável legal pela participação das encarceradas em atividades que envolvem pesquisas. Nesse caso, com a prerrogativa de manter a privacidade das mulheres encarceradas, a instituição prisional exigia anonimato, autorizando as mesmas a participarem de modo voluntário, mas proibindo qualquer tipo de registro que pudesse identificar essas mulheres fora do presídio. 
Deste modo, as participantes foram orientadas quanto às exigências citadas e ao aceitaram participar da pesquisa, a pesquisadora ia transcrevendo os depoimentos, oportunizando leitura das transcrições para que as entrevistadas pudessem ouvir e demonstrar (des)acordo. Com a garantia do sigilo e o anonimato, as falas eram identificadas por códigos alfanuméricos de " $\mathrm{M} 1$ " a "M40", observando-se a sequência de realização das entrevistas.

A pesquisa foi aprovada pelo Comitê de Ética e Pesquisa da Universidade Federal Fluminense (CEP/UFF), sob protocolo n.응 696.795/2014 e autorizada pela Secretaria de Administração Penitenciária do Rio de Janeiro, pelo processo E-2108753/2014, atendendo à Resolução n. 0 466/2012 do Conselho Nacional de Saúde (CNS), que dispõe sobre pesquisa com seres humanos.

Para analisar os resultados, optou-se pela análise de conteúdo na modalidade temática ${ }^{(14)}$, com o fim de obter informações que expressassem aproximação com a realidade proferida pelas entrevistadas.

\section{Resultados}

A partir das falas das mulheres e após leitura dos depoimentos, foi possível identificar, em relação ao acesso à saúde, duas categorias temáticas: 1) O acesso das mulheres aos serviços de saúde antes da prisão; 2) O acesso das mulheres aos serviços de saúde durante o encarceramento.

\section{O acesso das mulheres aos serviços de saúde antes da prisão}

Antes do período de encarceramento, pode-se observar que os relatos das mulheres apontam que a procura pelos serviços de saúde ocorre em situações esporádicas a partir dos episódios de doença ou de uma determinada queixa. Vide a fala de uma participante ao sinalizar que sua ida ao serviço de saúde ocorria:

"Quando tinha algum problema" (M25).

Ou observe o depoimento de outra mulher, ao destacar que somente procurava assistência em saúde, quando as suas condições eram precárias:
"Só em casos precários eu procurava atendimento mesmo" (M8).

Ainda foi possível identificar a existência de mulheres que mesmo tendo relatos de morbidades pregressas, como hipertensão e diabetes, não tinham por hábito acessar os serviços de saúde numa perspectiva de acompanhamento da saúde, de forma negligenciada à sua condição de saúde física, psicológica, emocional, como pode ser exemplificado a partir do relato:

"Nunca fui ao serviço de saúde em liberdade, não buscava atendimento, não tinha esse hábito" (M37).

Essa negativa quanto ao acesso aos serviços de saúde (ou falta dele) pode ser percebida ainda por meio de informações demonstradas a partir de respostas direcionadas a não realização de questões específicas ou de determinado procedimento como, por exemplo, o exame preventivo do câncer de colo uterino. Assim, os depoimentos que seguem demonstram que até mesmo para a realização de um determinado procedimento essas entrevistadas não conseguiam acessar os serviços de saúde que atendessem suas necessidades.

"Nunca fiz exame ginecológico, pois não consegui realizar" (M16).

"Nunca fiz um preventivo quando estava em liberdade, era difícil marcar, era difícil conseguir o médico" (M21).

Tais falas demonstram que as mulheres esbarravam em contratempos para efetivar 0 acesso aos serviços de saúde quando em liberdade, que podem nesse ponto serem inferidos pelo precário quantitativo de profissionais, pela demora relacionada à marcação de procedimentos ou atendimentos, ou ainda pela própria dificuldade econômica que indiretamente dialoga com a possível dificuldade de locomoção até a instituição. Assim, se reforça a ideia de que mesmo tendo conhecimento da necessidade de acessar ações individualizadas, sob aspecto de prontoatendimento ou ainda com viés curativo, essas mulheres perpassam por dificuldades para garantir atenção integral à saúde. 


\section{O acesso das mulheres aos serviços de saúde durante o encarceramento}

$\mathrm{Na}$ instituição prisional estudada, as mulheres cumprem pena em sistema fechado, logo, não possuem livre acesso aos serviços de saúde que estejam fora do complexo penitenciário.

A assistência à saúde das mulheres durante o período de encarceramento é realizada, na maioria das vezes, no ambulatório da unidade prisional. As ações realizadas encontram-se distantes de uma atenção integral conforme preconizado pela política vigente. Pois, o acesso ao ambulatório de atendimentos individuais ocorre, ou a partir de solicitações próprias através de bilhetes que são encaminhados aos profissionais de saúde, ou quando a segurança solicita atendimento específico para alguma mulher encarcerada. Interessante apontar que esta última situação acontece somente quando as mulheres apresentam algum problema de saúde que está interferindo no cotidiano da penitenciária. A partir da questão "Você utiliza o serviço de saúde desta penitenciária?", pode-se observar depoimentos que sinalizam processos de dificuldades vivenciados por resistências, tensões ou ainda por interferências estruturais.

"Não, quase não venho aqui (ambulatório prisional), quando mando bilhete não me chamam" (M1).

“Não, não consigo" (M17).

"Aqui é ruim de água (ambulatório prisional), este dia, eu estava cheia de machucado e ninguém me chamou" (M20).

Tais resistências podem ser originárias, tanto por parte dos profissionais da segurança, quanto pelas próprias entrevistadas. Vide exemplo a partir da seguinte fala:

"Tomo remédio para não precisar ir ao serviço de saúde. Tenho medo do SOE (Serviço de Operações Especiais), sou desaforada, tudo que me traga problema eu evito" (M13).

"Sim, mas é difícil. Este ambulatório não serve, preciso de ginecologista, mas não tem" (M12).
"Sim, várias vezes. Por causa dos remédios controlados" (M2).

Com relação à necessidade das mulheres serem referenciadas pelos profissionais do sistema penal para algum serviço fora do ambulatório prisional, ocorrem solicitações de autorização à direção e realização de agendamentos na Coordenação de Saúde do Sistema Penitenciário do Rio de Janeiro, Brasil. É importante destacar que, o transporte das pessoas privadas de liberdade para atendimentos de saúde na rede pública é realizado pelo SOE (Serviço de Operações Especiais). Quando existe a necessidade de serviços hospitalares/internações, as mulheres também são transferidas com escolta, de ambulância da SEAP-RJ para instituições da rede fora do complexo penitenciário. No caso da necessidade de serviço de urgência/emergência, as mulheres são encaminhadas à Unidade de Pronto Atendimento (UPA) dentro do próprio complexo penitenciário da SEAP-RJ e, se necessário, são encaminhadas para a rede pública.

Deste modo, conforme visto nos depoimentos acima, o serviço de saúde da penitenciária estudada ainda é caracterizado por uma atenção individualizada, curativista ou realizado por livre demanda; e perpassado por dificuldades relacionadas ao acesso às atividades relacionadas à saúde.

No entanto, mesmo que seja perpassado por dificuldades e que em alguma medida ainda seja desqualificado por algumas entrevistadas, pode-se observar que algumas mulheres reconhecem que têm o direito de acesso à saúde garantido por lei e, além disso, provocam situações para que seja efetivado. As entrevistadas demonstraram reconhecimento de seus direitos e essa questão pode ser expressa nas seguintes falas:

"Estou presa, mas os meus direitos de cidadania e saúde têm de permanecer. Eu já estou pagando o que eu devo, preciso sair daqui viva e com saúde" (M2).

"Fiz consulta ginecológica, fui ao dentista, psicólogo e ao assistente social, tudo por solicitação judicial" (M18).

"A gente paga o que deve para justiça, mas para sociedade a cadeia é eterna, nossos direitos não são garantidos, a saúde é um direito de todos" (M15). 
Mesmo que tenham sido contraditórios, os depoimentos acima apresentam viabilidade para destacar que o acesso aos serviços de saúde durante o encarceramento pode ser permeado por restrições, mas em alguma medida, efetivado.

\section{Discussão}

O entendimento das entrevistadas sobre as questões de saúde ainda se encontra relacionado à ausência de doença, não existindo a percepção de que os serviços de saúde deveriam desenvolver ações relacionadas à promoção da saúde. Deste modo, pode ser observada a partir do campo empírico, falas relacionadas à ausência de procura pelos serviços de saúde e a banalização da necessidade de realização de exames com vistas à prevenção de doenças.

Como as necessidades de saúde distribuem-se de forma desigual entre as pessoas de diferentes classes sociais e são mais evidentes nas classes de menor rendimento, geralmente, as mulheres encarceradas, que são provenientes desses grupos socialmente marginalizados, vivenciam processos de dificuldade ou deficiência de acesso aos serviços de saúde ${ }^{(15)}$.

Com este estudo, pode-se observar que tanto o acesso aos serviços de saúde antes do encarceramento quanto após o mesmo, ainda encontra-se focalizado ao tratamento individual e distanciado dos aspectos de promoção, prevenção e recuperação da saúde, conforme determina a política pública de saúde específica para as mulheres privadas de liberdade ${ }^{(13-16)}$.

A partir das entrevistas realizadas com as mulheres encarceradas, constatou-se a presença de dificuldades para acessar os serviços de saúde (tanto antes quanto após a prisão), similares às informações compartilhadas em estudo(6), que apresenta as dificuldades de acesso a partir das dimensões estrutural (financiamento e cobertura), relacional (compreensão do processo saúdedoença) e operacional (organização dos serviços). Ou seja, as participantes desse estudo, sobretudo no que diz respeito ao período em que se encontravam antes da prisão, enfrentavam dificuldades de cobertura e agendamentos de serviços. O que não descarta a possibilidade de ocorrer lapsos temporais por parte destas mulheres, retardando a demanda pelos serviços de saúde.

Assim, as dificuldades de acesso à saúde ficaram evidentes tanto para a mulher que estava fora do sistema prisional quanto para a mulher encarcerada ${ }^{(17)}$, contrariando o que preconizam as Políticas Públicas de Saúde frente às diretrizes e princípios que regem o SUS ${ }^{(13)}$. Vide a existência de mulheres que necessitam de mandato judicial para a conquista do acesso ao serviço de saúde.

No entanto, com relação ao período de encarceramento no sistema prisional, mesmo diante de serviços de saúde que apresentam restrições para prover o atendimento necessário e adequado às mulheres privadas de liberdade (deficitário quantitativo de recursos humanos, equipamentos, medicamentos e espaço físico), pode-se observar de modo restrito, contraditório e sem possibilidade de generalizações, que em alguma medida, existem ações que oportunizam o acesso aos serviços de saúde ${ }^{(18-19)}$.

Ao estar de acordo que o direito ao acesso à saúde é uma das prerrogativas da Constituição Brasileira que deve ser garantido via políticas públicas de saúde, inclusive àquelas relacionadas ao sistema prisional do país, entende-se que este direito não deva ser negligenciado, sobretudo para a população feminina encarcerada. Pois, existe a necessidade de ressaltar que essa população ao prosseguir com o entendimento relacionado ao conceito de saúde como ausência de doenças, além de apresentar especificidades relacionadas ao gênero, se encontra em situações de vulnerabilidades que interferem negativamente nas condições de saúde.

Considerando que o sistema prisional ainda favorece a ocorrência de agravos à saúde, debilidades funcionais e restrições de atividades $^{(21)}$, a garantia do direito ao acesso à saúde das mulheres privadas de liberdade deve ser fomentada tendo em vista a necessidade de preservação das condições de saúde e a garantia de uma atenção integral à saúde. Ou seja, o sistema prisional deve ter um foco além de sua dimensão curativista, abordando o cuidado com vistas às ações de promoção, prevenção de agravos, tratamento de doenças, recuperação da saúde e melhoria da qualidade de vida. Pois, quando este direito é negado dentro das prisões, torna-se um problema para toda sociedade ${ }^{(22)}$.

Mesmo diante das contribuições, cabe 
apresentar algumas limitações relacionadas a este estudo: burocracia estabelecida pelo sistema prisional para efetivar a autorização para o início da pesquisa; limitação de tempo disponibilizado pela instituição para a coleta de informações; proibição do uso de gravações de áudio e filmagem, fotografias e a reprodução de qualquer documento no interior do presídio; limitação do quantitativo diário de entrevistas; acompanhamento das mulheres por uma agente de segurança na entrada e saída da realização da entrevista.

\section{Conclusão}

Analisar sobre a (não) garantia de acesso aos serviços de saúde antes e durante o período de encarceramento contribui para observar como tem sido o processo de (des)qualificação das atividades nessas instituições. Mostra-se de suma importância, tendo em vista que o atendimento dispensado à mulher encarcerada deve contemplar as necessidades e demandas reais com vistas à consolidação de uma atenção integral à saúde desta população. Além da necessidade de reorientar ou implementar a dinâmica de algumas atividades relacionadas aos serviços de saúde de maneira geral.

As falas das mulheres presas deixam claro que esta população encontra dificuldades de acesso aos serviços de saúde dentro e fora do presídio, demonstrando que se encontram em processo de exclusão, mesmo antes da reclusão. No entanto, é válido destacar que, em alguma medida durante a prisão, passam a acessar serviços que antes do encarceramento não conseguiam e até mesmo não validavam sua importância. Mesmo assim, sobretudo as unidades prisionais, deveriam proporcionar às mulheres encarceradas a continuidade desse acesso para além de sua entrada no sistema para atendimentos individuais. Ou seja, deveriam também garantir a realização de exames, finalização de tratamentos e acompanhamentos integrais de morbidades crônico-degenerativas.

Mesmo que tenha sido destacada uma realização pontual de atividades relacionadas à saúde, torna-se interessante compartilhar que essas ações deveriam fazer parte de um contexto ampliado diante da complexidade e vulnerabilidade que envolve a vida da mulher durante a prisão.
Em geral, torna-se imprescindível que os serviços de saúde, tanto dentro quanto fora do sistema penitenciário, promovam ambientes mais saudáveis, ações resolutivas e facilitem o acesso das mulheres à uma assistência integral nas diversas etapas da vida.

\section{Referências}

1. Ministério da Justiça (BR). Lei n. ${ }^{\circ} 7.210$, de 11 de julho de 1984, dispõe da Lei de execução penal brasileira. Brasília: Ministério da Justiça; 1984.

2. Moraes A. Constituição da República Federativa do Brasileira de 05 de outubro de 1988. 43ำ edição. São Paulo: Editora Atlas; 2017.

3. Carvalho G. A saúde pública no Brasil. Estud. Av. 2013; 27(78): 7-26.

4. Campos RTO, Ferrer AL, Gama CAP, Campos GWS, Trapé TL, Dantas DV. Avaliação da qualidade do acesso na atenção primária de uma grande cidade brasileira na perspectiva dos usuários. Saúde Debate. 2014; 38 (esp): 252-64.

5. Sanchez RM, Ciconelli RM. Conceitos de acesso à saúde. Rev Panam Salud Publica. 2012; 31(3): 260-8.

6. Viegas APB, Carmo RF, Luz ZMP. Fatores que influenciam o acesso aos serviços de saúde na visão de profissionais e usuários de uma unidade básica de referência. Saúde Soc. 2015; 24(1):100-12.

7. Stopa SR, Malta DC, Monteiro CN, Szwarcwald CL, Goldbaum M. \& Cesar CLG. Use of and access to health services in Brazil, 2013 National Health Survey. Rev. Saúde Pública. 2017; 51(suppl. 1):1S-10S.

8. Silva JO, Valente GSC. O enfermeiro de saúde coletiva no tratamento e acompanhamento do idoso soropositivo. Rev Enferm Atual. 2017; 20(82): 19-26. 
9. Assis MMA, Jesus WLAD. Acesso aos serviços de saúde: abordagens, conceitos, políticas e modelo de análise. Ciênc. Saúde Coletiva. 2012; 17(11):2865-75.

10. Ministério da Justiça (BR). Portaria Interministerial n. 0 1.777, de 9 de setembro de 2003. Dispõe sobre Plano Nacional de Saúde do Sistema Penitenciário. Brasília: Ministério da Justiça; 2003.

11. Lermen HS, Gil BL, Cúnico SD, Jesus LO. Saúde no cárcere: análise das políticas sociais de saúde voltadas à população prisional brasileira. Physis. 2015; 25(3): 905-24.

12. Ministério da Justiça (BR). Portaria Interministerial $n$. o 1, de 2 de janeiro de 2014. Institui a Política Nacional de Atenção Integral à Saúde das Pessoas Privadas de Liberdade no Sistema Prisional. Ministério da Justiça; 2014.

13. Ministério da Saúde (BR). Portaria Interministerial n.o 210, de 16 de janeiro de 2014. Institui a Política Nacional de Atenção às Mulheres em Situação de Privação de Liberdade e Egressas do Sistema Prisional. Ministério da Saúde; 2014.

14. Bardin, L. Análise de Conteúdo. São Paulo: Edições 70; 2011.

15. Nicolau AIO, Pinheiro AKB. Condicionantes sociodemográficos e sexuais do conhecimento, atitude e prática de presidiárias quanto ao uso de preservativos. Texto \& Contexto Enferm. 2012; 21(3):58190.

16. Fertonani HP, Pires DEP, Biff D, Scherer MDA. Modelo assistencial em saúde: conceitos e desafios para a atenção básica brasileira. Cência \& Saúde Coletiva. 2015; 20(6):186978.

17. Miltão LP, Kruno RB. Vivendo a gestação dentro de um sistema prisional. Saúde (Santa Maria). 2014; 40(1):75-84.

18. Barbosa ML, Celino SDM, Oliveira LV, Pedraza DF, Costa GMC. Atenção básica à saúde de apenados no sistema penitenciário: subsídios para a atuação da enfermagem. Esc. Anna Nery. 2014; 18(4):586-92.

19. Almeida PRC, Soares RSC, Coura AS, Cavalcanti AL, Dutra MOM, Lima TMA. Condição de Saúde de Mulheres Privadas de Liberdade: uma revisão integrativa. Rev. Bras. Ciênc. Saúde. 2015; 19(1):73-80.

20. Silva M. Saúde Penitenciária no Brasil: plano e política. Brasília: Verbena; 2015.

21. Bergh BJVD, Gatherer A, Fraser, A, Moller, L. Imprisonment and women's health: concerns about gender sensitivity, human rights and public health. Bulletin of the World Health Organization. 2011; 89(9):689-94.

22. Damas FB. Assistência e condições de saúde nas prisões de Santa Catarina, Brasil. Rev. Saúde Públ. Santa Cat. 2012; 5(3):6-22. 